\title{
Passivation of InP solar cells using large area hexagonal-BN
}

\section{layers}

Vidur Raj ${ }^{1,6 凶}$, Dipankar Chugh ${ }^{1,6}$, Lachlan E. Black², M. M. Shehata ${ }^{2}$, Li Li $\mathbb{D}^{3}$, Felipe Kremer ${ }^{4}$, Daniel H. Macdonald ${ }^{2}$, Hark Hoe Tan ${ }^{1,5 \times}$ and Chennupati Jagadish ${ }^{1,5}$

Surface passivation is crucial for many high-performance solid-state devices, especially solar cells. It has been proposed that $2 \mathrm{D}$ hexagonal boron nitride ( $\mathrm{hBN}$ ) films can provide near-ideal passivation due to their wide bandgap, lack of dangling bonds, high dielectric constant, and easy transferability to a range of substrates without disturbing their bulk properties. However, so far, the passivation of hBN has been studied for small areas, mainly because of its small sizes. Here, we report the passivation characteristics of wafer-scale, few monolayers thick, hBN grown by metalorganic chemical vapor deposition. Using a recently reported ITO/i-InP/ $\mathrm{p}^{+}-\mathrm{InP}$ solar cell structure, we show a significant improvement in solar cell performance utilizing a few monolayers of hBN as the passivation layer. Interface defect density (at the hBN/i-InP) calculated using $C-V$ measurement was $2 \times 10^{12} \mathrm{eV}^{-1} \mathrm{~cm}^{-2}$ and was found comparable to several previously reported passivation layers. Thus, hBN may, in the future, be a possible candidate to achieve high-quality passivation. hBN-based passivation layers can mainly be useful in cases where the growth of lattice-matched passivation layers is complicated, as in the case of thin-film vapor-liquid-solid and close-spaced vapor transport-based III-V semiconductor growth techniques.

npj 2D Materials and Applications (2021)5:12; https://doi.org/10.1038/s41699-020-00192-y

\section{INTRODUCTION}

At the semiconductor surface, abrupt termination of the lattice can lead to defect states (called surface defect states) within the semiconductor bandgap. These surface defect states can significantly impair the working of semiconductor devices, such as solar cells. Passivation is a method for reducing recombination through these surface defect states and is one of the most fundamental requirements for achieving high-efficiency solar cells. In conventional III-V solar cells, epitaxially grown, lattice-matched III-V materials allowed very high-quality passivation ${ }^{1}$. However, most of these lattice-matched wide bandgap III-Vs are only available for GaAs. Although high-efficiency InP solar cells have been realized using lattice-matched InGaAs and InAlAs passivation layers, when used at the front, both of these materials have a sizeable parasitic absorption due to low bandgaps ${ }^{2-5}$. The lack of a wide bandgap passivation layer for InP has significantly impeded its development compared to that of GaAs solar cells ${ }^{1}$.

Other than epitaxial passivation layers, a substantial amount of work has also been done to find suitable non-epitaxial passivation layers (mainly high $k$-dielectric), including but not limited to $\mathrm{Al}_{2} \mathrm{O}_{3}$, $\mathrm{Gd}_{2} \mathrm{O}_{3}, \mathrm{LaF}_{3}, \mathrm{ZnO}, \mathrm{ZnS}, \mathrm{GaS}, \mathrm{SiO}_{2}, \mathrm{MgO}, \mathrm{Ta}_{2} \mathrm{O}_{3}, \mathrm{PO}_{\mathrm{x}}, \mathrm{Si}_{3} \mathrm{~N}_{\mathrm{x}}$, etc. ${ }^{1,6-10}$. Many of these dielectric materials have been shown to function as suitable passivation layers; however, in most cases, controlled and reproducible formation of high quality and defect-free passivation layer remains a challenging task ${ }^{11}$. Also, most of the work is reported for III-V transistors with very few reports on highefficiency III-V solar cells, using dielectric passivation.

On the other hand, pristine 2D materials, in particular, hexagonal boron nitride ( $\mathrm{hBN}$ ) possess many desirable attributes, which makes them an ideal passivation layer. For instance, hBN and other 2D materials lack dangling bond and can be transferred to a range of substrates without disturbing its bulk properties ${ }^{11,12}$. Also, hBN has a wide bandgap, high chemical and thermal stability, and high dielectric constant $(\sim 2.4)$, which makes it ideal for surface passivation ${ }^{13}$. So far, the most pristine form of hBN is obtained through mechanical exfoliation from bulk crystals; however, the lateral size of exfoliated flakes is limited to only a few tens of microns, which severely limits the scalability and large area application of hBN. Therefore, new methods such as chemical vapor deposition (CVD), metalorganic chemical vapor deposition (MOCVD), and physical vapor deposition (e.g., DC magnetron sputtering) are being investigated to achieve large area $\mathrm{hBN}^{11,14}$.

hBN has recently been used as a passivation layer in silicon, GaAs, chalcogenide, organic, and 2D transition metal chalcogenides-based solar cells ${ }^{13,15-19}$. However, in most of these cases, hBN used were very small in size, which is not practical for real solar cells application. In this study, we extend the application of hBN films for the passivation of InP-based solar cells. Centimeter-size MOCVD-grown hBN films were transferred onto InP to study their passivation effect. We show that MOCVD-grown $\mathrm{hBN}$ can successfully passivate $\mathrm{i}-\mathrm{InP} / \mathrm{p}^{+}-\mathrm{InP}$ solar cells to reduce the dark current, as well as the reverse leakage current by orders of magnitude, leading to a better performance of the device. The cells passivated with $\mathrm{hBN}$ has a $V_{\mathrm{oc}}$ of $>800 \mathrm{mV}$, in comparison to $720 \mathrm{mV}$ for unpassivated $\mathrm{i}-\mathrm{InP} / \mathrm{p}^{+}-\mathrm{InP}$ device. We also investigate the effect of hBN film thicknesses and demonstrate that while a thinner hBN is required to achieve a sufficiently high $J_{\text {sc }}$ a thicker hBN film leads to better passivation and higher $V_{\text {oc }}$. External quantum efficiency (EQE) measurement of hBN passivated solar cells clearly show a significantly improved ( 20\%) response in the

\footnotetext{
'Department of Electronic Materials Engineering, Research School of Physics, The Australian National University, Canberra, ACT 2601, Australia. ${ }^{2}$ Research School of Electrical, Energy and Materials Engineering, ANU College of Engineering and Computer Science, The Australian National University, Canberra, ACT 2601, Australia. ${ }^{3}$ Australian National Fabrication Facility, Research School of Physics, The Australian National University, Canberra, ACT 2601, Australia. ${ }^{4}$ Centre for Advanced Microscopy, The Australian National University, Canberra, ACT 2601, Australia. ${ }^{5}$ ARC Centre of Excellence for Transformative Meta-Optical Systems, The Australian National University, Canberra, ACT 2601, Australia. ${ }^{6}$ These authors contributed equally: Vidur Raj, Dipankar Chugh. ${ }^{\circledR}$ email: vidur.raj@anu.edu.au; hoe.tan@anu.edu.au
} 
blue region of the solar spectrum, thereby confirming the passivation of the solar cells in the presence of hBN monolayers. The passivation due to hBN is further corroborated by double diode fitting and analysis of the dark IV curves. To gain further insight into the passivation effects of $\mathrm{hBN}$, ultraviolet photoelectron spectroscopy (UPS) and $C-V$ measurements were performed. Based on the analysis of our results, we postulate that the effectiveness of $h B N$ as a passivation layer is a combined effect of direct quantum mechanical tunneling and interface charge transfer. We also discuss the electron selectivity of hBN.

\section{RESULTS AND DISCUSSION}

hBN characterization

Before and after transferring hBN on InP for device fabrication, we performed some basic characterizations of our hBN samples, as shown in Fig. 1. Figure 1a shows an optical micrograph of $7 \mathrm{ML}$ hBN transferred onto $\mathrm{SiO}_{2} / \mathrm{Si}$ substrate. The hBN covered region can be easily identified due to the difference in its refractive index with air. The thickness of the hBN film was measured using atomic force microscope (AFM). Figure $1 \mathrm{~b}, \mathrm{c}$ respectively shows the thickness of 5 and $7 \mathrm{ML}$ hBN films measured along the step edge, using AFM linescans. Raman spectra of 5 and $7 \mathrm{ML}$ hBN films are shown in Fig. $1 \mathrm{~d}$. The peak at $1368 \mathrm{~cm}^{-1}$ corresponds to the $E_{2 \mathrm{~g}}$ vibrational mode of the $s p^{2}$ bonded boron nitride ${ }^{20}$, and its intensity increase with film thickness. Furthermore, prior to device fabrication, the successful transfer of hBN onto the InP substrate was also confirmed using $x$-ray photoelectron spectroscopy (XPS). Figure $1 \mathrm{e}, \mathrm{f}$ shows the spectrum corresponding of $\mathrm{B}-1 \mathrm{~s}$ and $\mathrm{N}-1 \mathrm{~s}$ core levels at 190.6 and $398.2 \mathrm{eV}$, respectively, which confirmed the presence $\mathrm{hBN}$ on $\ln \mathrm{P}$.
Solar cell IV and EQE characterization

Figure 2 shows a 3D schematic and the cross-sectional transmission electron microscopy (TEM) images of the solar cell. To assess the performance of hBN as a passivation layer, IV (light and dark) and quantum efficiency measurements were performed on $\ln P$ solar cells with and without hBN. For both 5 and $7 \mathrm{ML} h B N$ passivated samples, we fabricated three devices, and the results were highly reproducible. Figure 3 a shows IV characteristics of the hBN-InP solar cell @ 1 sun. It is quite evident from the figure that the introduction of hBN significantly improves both the $V_{\mathrm{oc}}$ and $J_{\mathrm{sc}}$ of the solar cell, while also improving its fill factor. The maximum efficiency of $17.2 \%$ was obtained for $5 \mathrm{ML} \mathrm{hBN}$ with a $V_{\text {oc }}$ of $0.78 \mathrm{~V}$, $J_{\text {sc }}$ of $29.4 \mathrm{~mA} \mathrm{~cm}^{-2}$ and a FF of $75.2 \%$. The efficiency of InP solar cell with $7 \mathrm{ML} h B N$ was measured to be $15.7 \%$ with a $V_{\text {oc }} J_{\text {sc }}$ and FF of $0.8 \mathrm{~V}, 27.1 \mathrm{~mA} \mathrm{~cm}^{-2}$, and $72.1 \%$, respectively. This clearly shows that a thicker hBN gives a slightly higher $V_{o c}$ but significantly lower $J_{\mathrm{sc}}$ suggesting that the hBN must not be too thick to achieve optimum performance of the device. In comparison to both 5 and $7 \mathrm{ML}$ passivated InP solar cells, the efficiency of unpassivated ITO/i-InP/p-InP remains significantly lower at $11.5 \%$ with a $V_{\text {oc }}$ of $0.72 \mathrm{~V}, J_{\text {sc }}$ of $27.4 \mathrm{~mA} \mathrm{~cm}^{-2}$, and a FF of $58.6 \%$. Here, we would like to mention that for the MOCVDgrown hBN films, it has been shown that for the continuous coverage over the entire 2 in. sapphire substrate, a minimum growth time of $1 \mathrm{~h}$ was necessary ${ }^{20}$. This growth duration resulted in hBN films with a thickness of $5 \mathrm{ML}$. Hence in the present study, hBN films thinner than $5 \mathrm{ML}$ could not be obtained. Nonetheless, in the future, it will be exciting to see if monolayers $<5 \mathrm{ML}$ can also provide sufficiently high passivation to achieve high efficiency. As expected, the lower $V_{\text {oc }}$ and $J_{\text {sc }}$ for unpassivated ITO/i-InP/p-InP solar cells are mainly due to interface recombination and are consistent with previously reported results ${ }^{1,5}$.
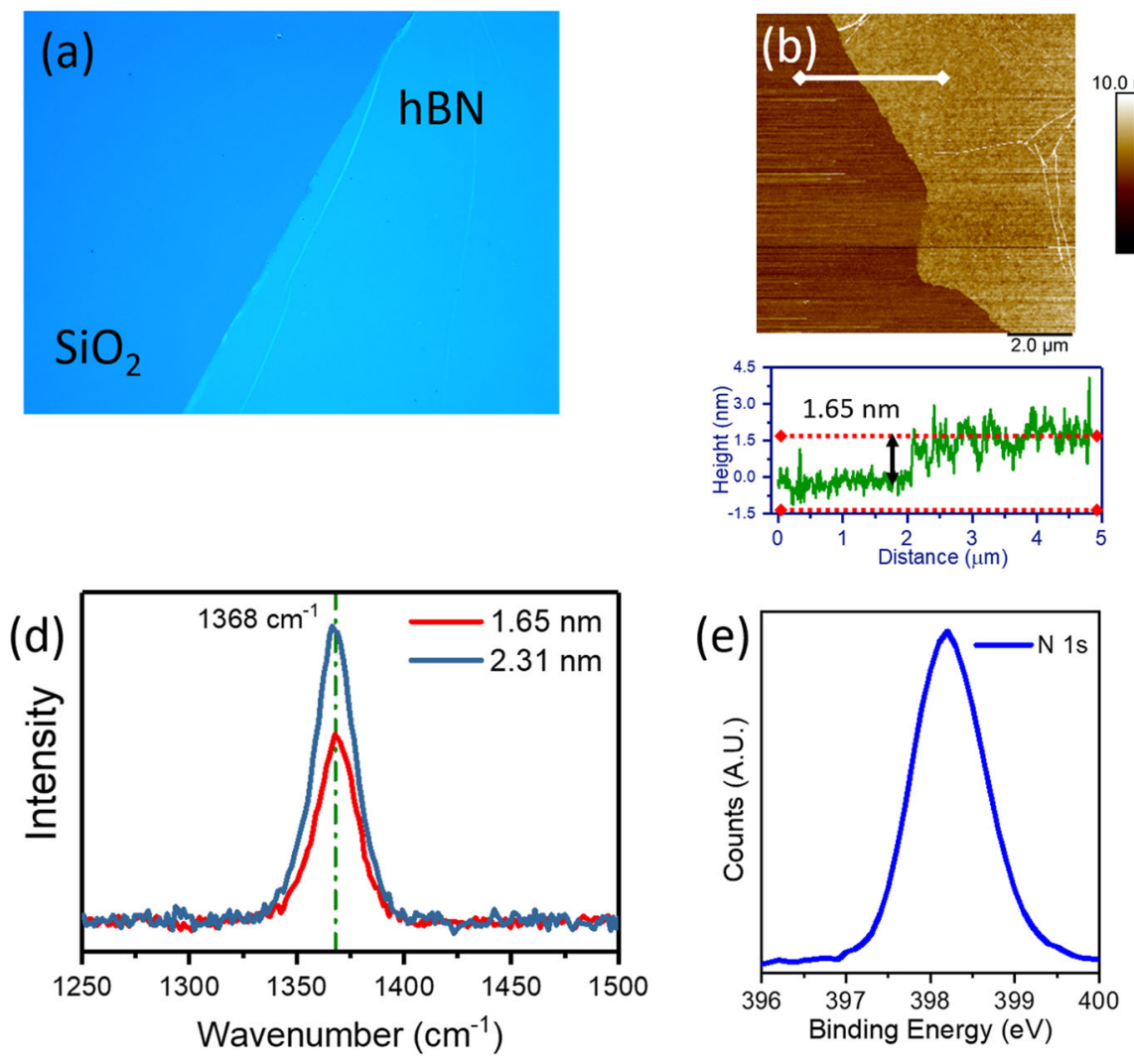
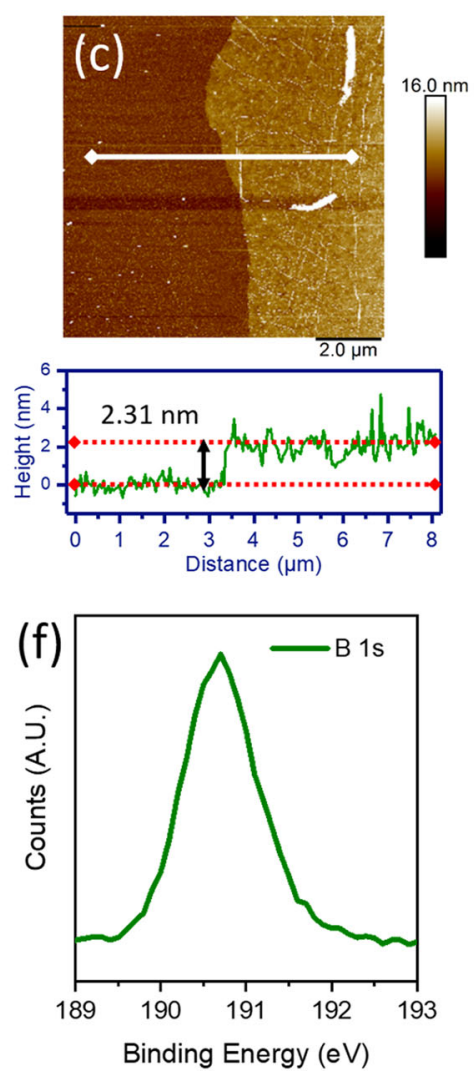

Fig. 1 Characterization of 2D hBN thin film. a Optical micrograph of hBN monolayer transferred onto a $\mathrm{SiO}_{2} / \mathrm{Si}$ substrate for $\mathrm{AFM}$ and Raman characterization. AFM results of $\mathbf{b} 5 \mathrm{ML}$ and $\mathbf{c} 7 \mathrm{ML} \mathrm{hBN}$. The scanned height variation of the film is shown below the AFM image. $\mathbf{d}$ Thicknessdependent Raman intensity of the hBN layer. XPS spectra of e B-1s and $\mathbf{f} \mathrm{N}-1 \mathrm{~s}$ of the hBN layer transferred onto InP. 
(a)
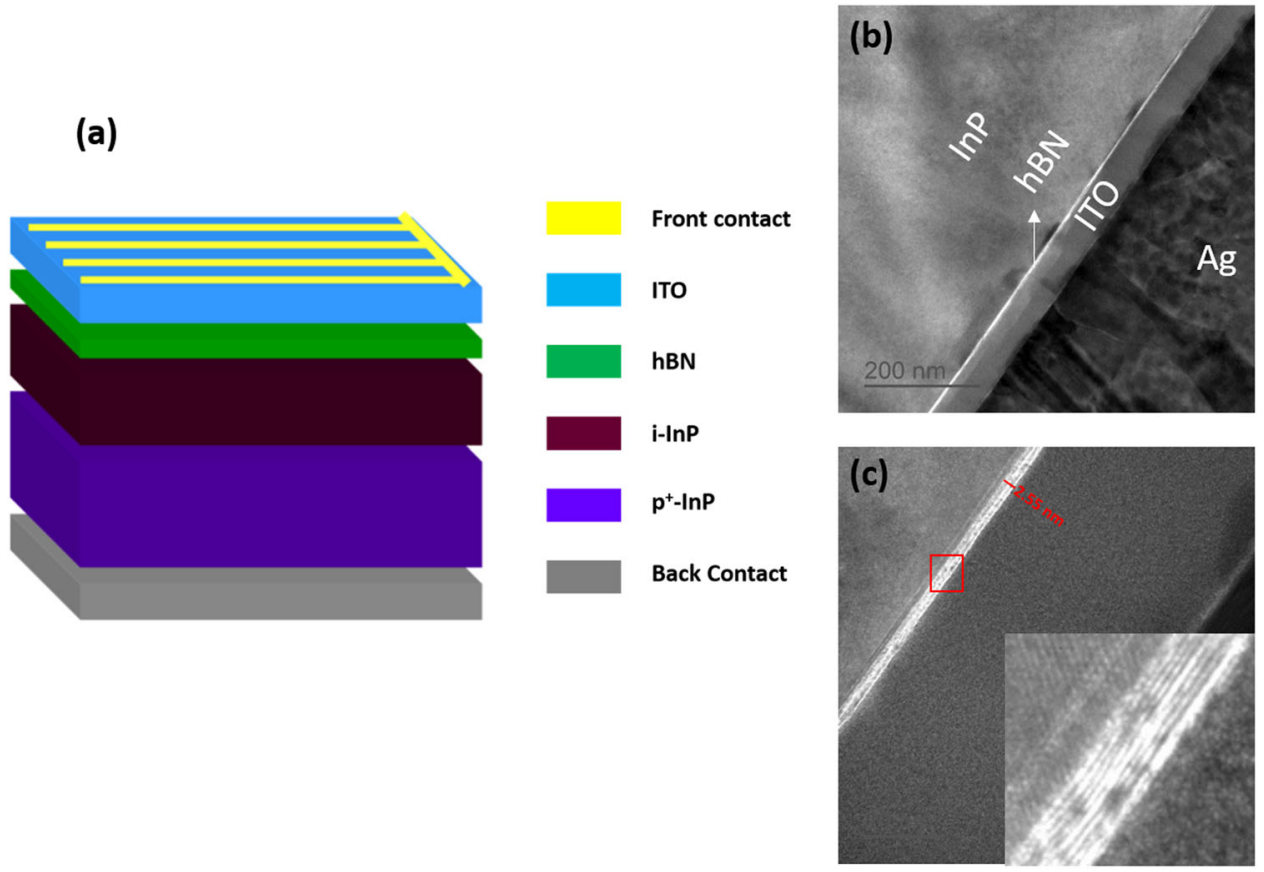

Fig. 2 3D schematic and TEM of device structure. a A schematic showing different layers of the solar cell. b A cross-sectional transmission electron microscope (TEM) image of the solar cell showing the different layers (scale bar: $200 \mathrm{~nm}$ ). c A magnified TEM image showing 2.55-nm thick hBN sandwiched between ITO and i-InP (scale bar: $20 \mathrm{~nm}$ ). Inset shows the layered structure of hBN.
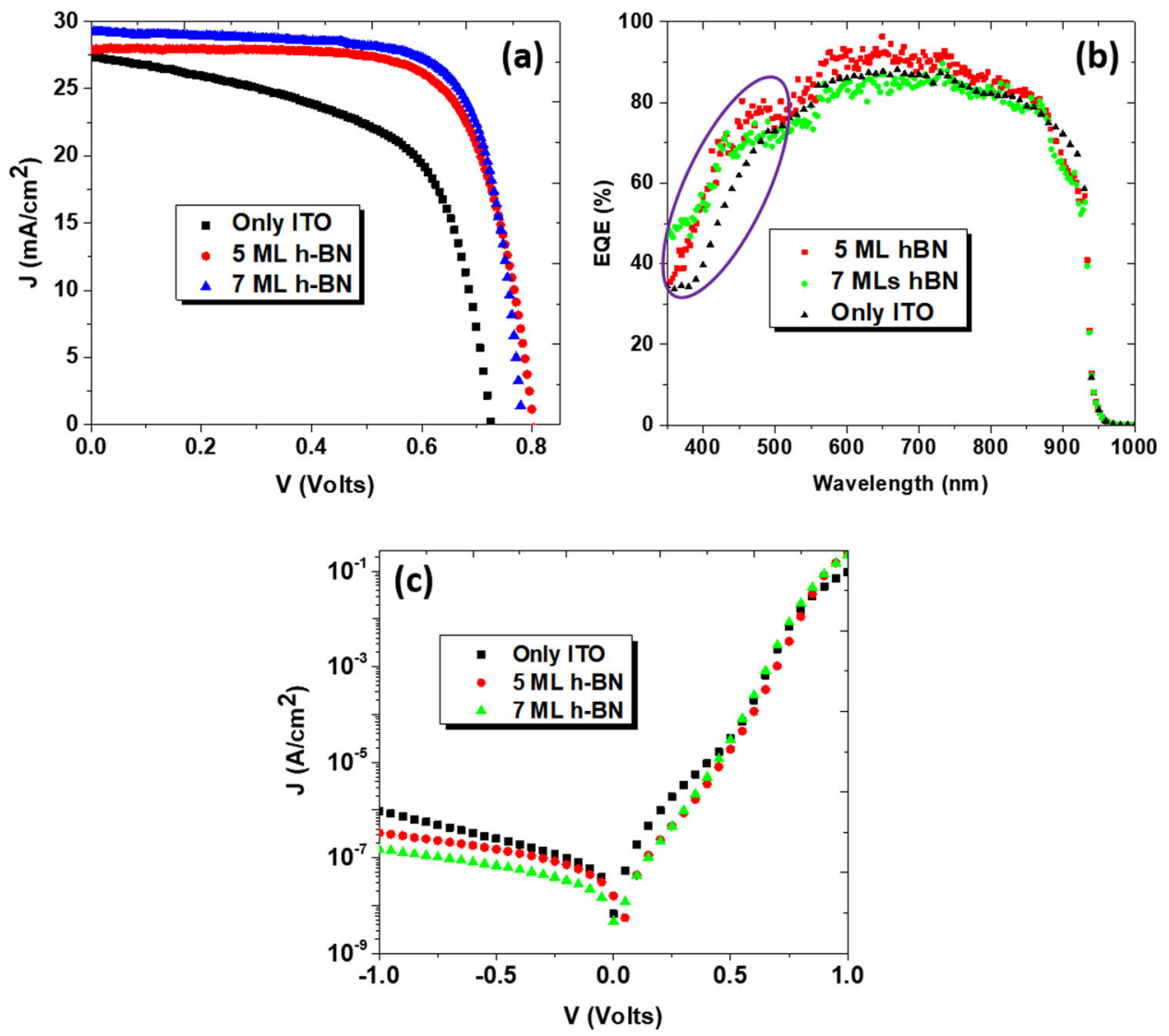

Fig. 3 IV and EQE of solar cells. Comparative a IV @ 1 sun, b EQE, and c dark IV for the solar cells with and without hBN layer. 
The EQE of a solar cell is a function of wavelength and is a direct measure of its $J_{\text {sc }}$. It can be written as a ratio of the number of photogenerated electrons collected to the number of photons of a given wavelength or energy incident onto the device:

$$
\operatorname{EQE}(\lambda)=\frac{I_{\mathrm{ph}}}{e \cdot \Phi_{\mathrm{p}}}=\frac{I_{\mathrm{ph}}}{e}\left(\frac{h u}{P_{\mathrm{opt}}}\right)
$$

where, $I_{\mathrm{ph}}$ is the measured current in short-circuit condition when illuminated with light of frequency, $v$ and optical power, $P_{\mathrm{opt}} e$ is the electronic charge, $h$ is the Planck's constant, and $\Phi_{\mathrm{p}}$ is the photon flux. The EQE calculated is related to $J_{\mathrm{sc}}$ of a solar cell using Eq. (2).

$$
J_{\mathrm{sc}}(\mathrm{EQE})=\int_{\lambda 1}^{\lambda 2} \frac{q \lambda}{h c}\{\operatorname{EQE}(\lambda) \cdot \operatorname{AM} 1.5 \mathrm{G}(\lambda)\} \mathrm{d} \lambda
$$

where $J_{s c}$ is the short-circuit current calculated for EQE measured between $\lambda_{1}$ and $\lambda_{2}$. Using Eq. (2), the $J_{\text {sc }}$ calculated for the unpassivated device is $26.7 \mathrm{~mA} \mathrm{~cm}^{-2}$, whereas the $J_{\mathrm{sc}}$ calculated for $5 \mathrm{ML}$ and $7 \mathrm{ML}$ passivated samples are 28.9 and $27.1 \mathrm{~mA} \mathrm{~cm}^{-2}$, respectively. As expected, the calculated $J_{\text {sc }}$ is very close to the $J_{\text {sc }}$ measured using light generated IV curve shown in Fig. 3a.

Moreover, because the different wavelength of light is absorbed in different regions of the solar cell, wavelength-dependent EQE can provide information about optoelectronic losses in the different regions of the device. In particular, the smaller wavelength light is absorbed in the topmost part of the absorber layer, and the quantum efficiency in this regime reflects the losses due to surface recombination. Figure $3 \mathrm{~b}$ shows measured EQE as a function of wavelength. It can be seen that there is a significant improvement in the EQE of the solar cell after $5 \mathrm{ML} h B N$ passivation in the lower wavelength region, i.e., between 350 and $600 \mathrm{~nm}$. An improvement of $20 \%$ of EQE at the lower wavelength region for $5 \mathrm{ML}$ hBN passivated samples in comparison to the unpassivated samples, indicate a significant reduction in front surface recombination. Moreover, similar to $5 \mathrm{ML}$ passivated samples, $7 \mathrm{ML}$ passivated samples also show better EQE in the lower wavelength regime compared to unpassivated samples, and a slightly higher EQE near $350 \mathrm{~nm}$ in comparison to $5 \mathrm{ML}$ hBN passivated samples, suggesting slightly better passivation than $5 \mathrm{ML}$ passivated samples. However, its overall quantum efficiency is lower than that of $5 \mathrm{ML} h B N$ due to reduced tunneling (as discussed later), with increased hBN thickness leading to a lower charge carrier collection, and $J_{\mathrm{sc}}$ according to Eqs.(1) and (2).

Dark IV characteristics of a solar cell provide several useful information about device behavior in terms of recombination current, ideality factor, reverse leakage current, and series and shunt resistances. The dark experimental IV of a solar cell can be fitted almost accurately using Eq. (3) below to derive parameters such as $J_{01}, J_{02}, n_{1}, n_{2}, R_{\mathrm{s}}$ and $R_{\mathrm{sh}}$ (ref. ${ }^{10}$ ).

$J=J_{01} \exp \left[\frac{q\left(V-J R_{\mathrm{s}}\right)}{k T}\right]+J_{02} \exp \left[\frac{q\left(V-J R_{\mathrm{s}}\right)}{n_{2} k T}\right]+\frac{V-J R_{\mathrm{s}}}{R_{\text {Shunt }}}$

In Eq. (3), the parameters, $J_{01}$ and $J_{02}$, respectively, denote dark current due to diffusion and recombination in the depletion region, whereas, $n_{1}$ and $n_{2}$, denote the ideality factor of diodes, and $R_{\mathrm{s}}$ and $R_{\mathrm{sh}}$ denote the series and shunt resistances, respectively. Table 1 compares fitting parameters of three different samples, and it is quite apparent that for all the samples, $J_{01}$ is almost similar, which signifies that the recombination in bulk is identical in all the samples. However, $J_{02}$ is nearly an order of magnitude lower for hBN passivated samples compared to the unpassivated sample. This is primarily due to the passivation effect of hBN layer, which modifies the carrier transport across i-InP/ITO interface (as discussed later), and reduces the recombination in the depletion regions. Also, all the solar cells have very high series and low shunt resistance that is also reflected in the lower fill factor of the devices. Assuming a double diode model, the fill factor loss due to series $\left(\Delta \mathrm{FF}_{R_{\mathrm{s}}}\right)$ and shunt resistances $\left(\Delta \mathrm{FF}_{R_{\mathrm{sh}}}\right)$ can
Table 1. Dark IV fitting parameters for the three types of solar cells investigated in this study.

\begin{tabular}{llll}
\hline & Only ITO & $5 \mathrm{ML} \mathrm{hBN}$ & $7 \mathrm{ML} \mathrm{hBN}$ \\
\hline$J_{01}\left(\mathrm{~A} \mathrm{~cm}^{-2}\right)$ & $1.1 \times 10^{-17}$ & $2 \times 10^{-16}$ & $8 \times 10^{-16}$ \\
$J_{02}\left(\mathrm{~A} \mathrm{~cm}^{-2}\right)$ & $2.2 \times 10^{-8}$ & $1.2 \times 10^{-9}$ & $3 \times 10^{-9}$ \\
$n_{1}$ & 1 & 1 & 1 \\
$n_{2}$ & 2.3 & 2.1 & 2.3 \\
$R_{\mathrm{sh}}\left(\Omega \mathrm{cm}^{2}\right)$ & $1 \times 10^{3}$ & $6 \times 10^{4}$ & $3 \times 10^{4}$ \\
$R_{\mathrm{s}}\left(\Omega \mathrm{cm}^{2}\right)$ & 3.56 & 1.9 & 2.46 \\
\hline
\end{tabular}

be given by Eq. (4) (ref. ${ }^{21}$ ).

$$
\begin{aligned}
& \mathrm{FF}_{0}=\mathrm{FF}+\frac{J_{\mathrm{mpp}}^{2} R_{\mathrm{s}}}{V_{\mathrm{oc}} J_{\mathrm{sc}}}+\frac{\left(V_{\mathrm{mpp}}+J_{\mathrm{mpp}} R_{\mathrm{s}}\right)^{2}}{R_{\mathrm{sh}} V_{\mathrm{oc}} J_{\mathrm{sc}}} \\
& \Delta \mathrm{FF}_{R_{\mathrm{s}}}=\frac{J_{\mathrm{mpp}}^{2} R_{\mathrm{s}}}{V_{\mathrm{oc}} J_{\mathrm{sc}}} \\
& \Delta \mathrm{FF}_{R_{\mathrm{sh}}}=\frac{\left(V_{\mathrm{mpp}}+J_{\mathrm{mpp}} R_{\mathrm{s}}\right)^{2}}{R_{\mathrm{sh}} V_{\mathrm{oc}} J_{\mathrm{sc}}}
\end{aligned}
$$

In the above equation, $\mathrm{FF}_{0}$ denotes the fill factor of the resistance-free cell and $\Delta \mathrm{FF}_{R_{\mathrm{s}}}$ and $\Delta \mathrm{FF}_{R_{\mathrm{sh}}}$, respectively, denote the fill factor loss due to series and shunt resistances. Moreover, in addition to resistances, recombination in the depletion region can also lead to lower fill factor. To calculate loss due to depletion region recombination, we firstly need to calculate an upper limit of fill factor for the cell, which can be given using an analytical equation proposed by Green ${ }^{22}$.

$$
\mathrm{FF}_{J_{01}}=\frac{v_{\mathrm{oc}}-\ln \left(v_{\mathrm{oc}}+0.72\right)}{v_{\mathrm{oc}}+1}
$$

where

$v_{\mathrm{oc}}=\frac{q}{n k T} V_{\mathrm{oc}}$

Combining Eqs. (7) and (4), the fill factor loss due to recombination in the depletion region is simply the difference between $\mathrm{FF}_{\mathrm{J}_{01}}$ and $\mathrm{FF}_{0}$. Moreover, using Eq. 7, the upper limit of the fill factor $\left(\mathrm{FF}_{\mathrm{J01}}\right)$ for both passivated and unpasssivated samples come out to be $>85 \%$. For $5 \mathrm{ML}$ hBN passivated samples, the fill factor loss due to series and shunt resistance comes out to be $4.1 \%$ and $0.1 \%$, respectively, whereas the $J_{02}$ recombination loss is $\sim 6.3 \%$. For $7 \mathrm{ML}$ hBN passivated samples, with an increased thickness of hBN, series resistance increases slightly, leading a slightly higher series resistance loss. However, as expected, fill factor loss due to shunt resistance and $J_{02}$ recombination is almost similar to $5 \mathrm{ML}$ passivated samples. Most importantly, for unpasssivated samples, the most prominent fill factor loss mechanism is the recombination loss due to $J_{02}$ current, which is consistent with the results discussed earlier.

\section{UPS and band alignment}

To better understand the effect of hBN on the hBN/i-InP interface, we performed UPS measurements on ITO, i-InP, and hBN/i-InP. The UPS spectra for samples with and without hBN are plotted in Fig. $4 \mathrm{a}$, with binding energy (B.E.) on the $x$-axis and intensity on the $y$ axis. Before every measurement, the instrument was calibrated against a standard silver sample and the valence band maxima (VBM) was calculated by extrapolating the valence band contribution to the B.E. axis. The work function of the samples was calculated using the following equation:

$$
\phi=h v-\left(E_{\text {cut-off }}-E_{\text {Fermi }}\right)
$$

where $h v=21.2 \mathrm{eV}$ for He-I line, $E_{\text {cut-off }}$ is calculated by 
(a)

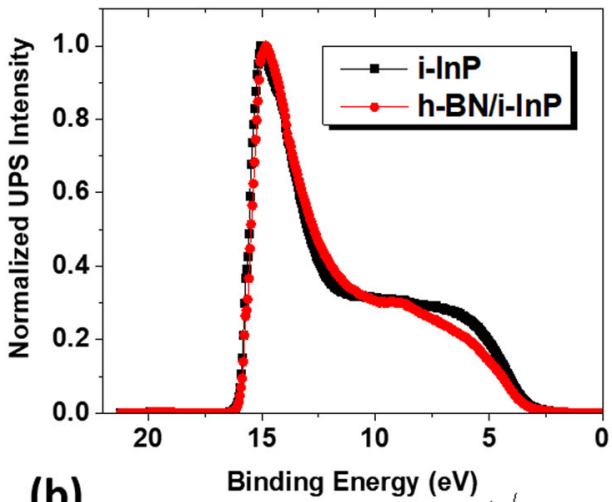

(c)
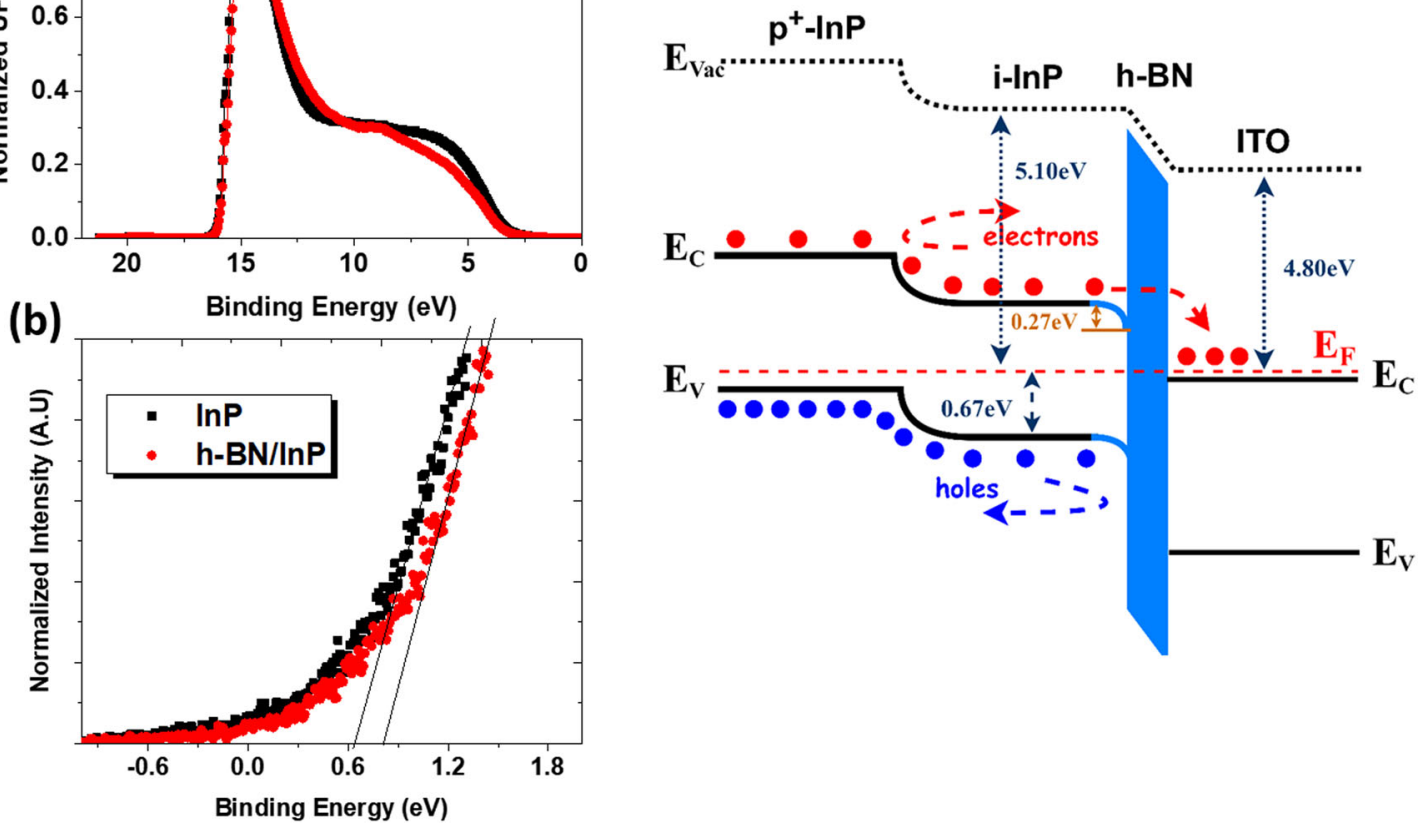

Fig. 4 UPS and band alignment of the solar cell. a Comparative UPS spectra of i-InP with and without hBN monolayer. b Magnified valence band contribution of the UPS spectra of $\mathrm{i}-\mathrm{InP}$ with and without hBN layer. c Band diagram drawn using UPS data to show the band bending at the interface of hBN/i-InP.

extrapolating the secondary electron onset to the $x$-axis, and $E_{\mathrm{Fermi}}$ is the corrected Fermi level. Because we calibrated the Fermi level before every measurement (using an Ag sample), for work function calculation, we assume $E_{\mathrm{Fermi}}=0$. Using Eq. (8), the work function of ITO and i-InP were calculated to be 4.8 and $5.1 \mathrm{eV}$, respectively. Surprisingly, there was no significant change in the work function of $\mathrm{i}-\mathrm{InP}$ before and after hBN transfer, as shown in Fig. 4a. However, there was a shift in VBM of i-InP after $h B N$ transfer. Figure $4 \mathrm{~b}$ shows the magnified valence band region of the $\mathrm{i}-\mathrm{InP}$, with and without hBN. The VBM of $\mathrm{i}-\mathrm{InP}$ moves by almost $0.27 \mathrm{eV}$ toward the Fermi level in the presence of $\mathrm{hBN}$. Based on UPS results, we draw a band diagram shown in Fig. 4c. It is quite evident in the band diagram that there is an accumulation of electrons at the hBN/i-InP interface, which has an important consequence toward reducing the interface recombination and increasing the selectivity of electrons, as discussed later in the passivation mechanisms section.

\section{Passivation mechanism}

To further corroborate the observed passivation at the $h B N / i-I n P$ interface, high-frequency $(100 \mathrm{kHz})$ capacitance-voltage $(C-V)$ measurements were performed on $\mathrm{Au} / \mathrm{ITO} / \mathrm{hBN} / \mathrm{i}-\mathrm{InP} / \mathrm{n}-\mathrm{InP}$ test structures, where ITO and Au were deposited through a shadow mask to form circular gate contacts. Interface state density $\left(D_{i t}\right)$ was extracted via the method of Terman (see Fig. 5) ${ }^{23}$, and similar energy-dependent interface state density was observed both 5 and $7 \mathrm{ML}$ hBN passivated samples, with a $D_{\text {it }}$ of $2-3 \times 10^{12} \mathrm{eV}^{-1} \mathrm{~cm}^{-2}$ at midgap. This compares favorably with reports for other passivating $3 \mathrm{D}$ thin films on $\operatorname{lnP}$, as compared in Table 2. However, unlike 3D passivation layers, the passivation in the case of $2 \mathrm{D} \mathrm{hBN}$ is not due to the passivation of dangling bonds. Based on previous reports, we postulate that $2 \mathrm{D} \mathrm{hBN}$ passivates the surface defects through the transfer of charges from

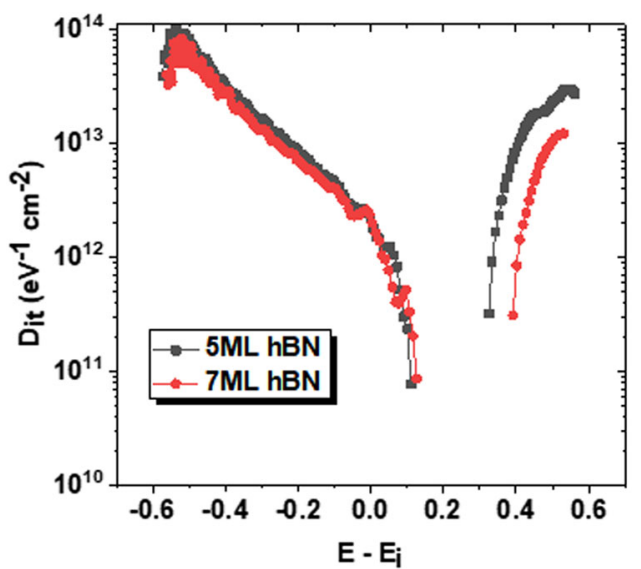

Fig. 5 Interface defect density at $\ln \mathrm{P} / \mathrm{hBN}$ interface. The energydependent interface state density extracted for the hBN films of different thickness from high-frequency $(100 \mathrm{kHz}) \mathrm{C}-V$ measurements. Note that the apparent dip/minima between 0 and $0.4 \mathrm{eV}$ is an expected artifact of the extraction method and should be ignored. $D_{\text {it }}$ at the intrinsic Fermi level $E_{\mathrm{i}}$ is $\sim 2 \times 10^{12} \mathrm{eV}^{-1} \mathrm{~cm}^{-2}$ in both cases, and slightly higher at midgap $\left(\sim 37 \mathrm{meV}<E_{\mathrm{i}}\right.$ for $\operatorname{InP}$ at room temperature).

surface defects states to hBN, which renders them electronically inactive and reduces electronically active interface defect density ${ }^{24}$.

Although $C-V$ measurement provides evidence of hBN passivation, it does not provide the reasoning for thickness-dependent hBN passivation, as evidenced in IV and EQE measurements. To further explain thickness-dependent hBN passivation and electron selectivity, we discuss the mechanism of charge carrier tunneling 
Table 2. Comparison of hBN passivation with other 3D passivation layers.

\begin{tabular}{lll}
\hline Passivation layer & Interface defect density $\left(\mathrm{eV}^{-1} \mathrm{~cm}^{-2}\right)$ & Reference \\
\hline $2 \mathrm{D} \mathrm{hBN}$ & $2 \times 10^{12}$ & Present work \\
$\mathrm{Al}_{2} \mathrm{O}_{3}$ & $2.6 \times 10^{12}$ & 25 \\
$\mathrm{HfO}_{2}$ & $5.4 \times 10^{12}$ & 25 \\
$\mathrm{TiO}_{2}$ & $1.6 \times 10^{12}$ & 26 \\
$\mathrm{SiO}_{2}$ & $5 \times 10^{12}$ & 27 \\
$\mathrm{SiN}_{\mathrm{x}}$ & $>1 \times 10^{12}$ & 28 \\
\hline
\end{tabular}

across hBN monolayers. Almost all quantum mechanical tunneling equation is an extension of standard Fowler-Nordheim (FN) tunneling equation, which in its simplest form is given by:

$$
J_{\mathrm{FN}}=A \cdot \frac{m_{\mathrm{ox}}^{*}}{\phi_{\mathrm{b}}} \cdot\left|F_{\mathrm{ox}}\right|^{2} \cdot \exp \left(-B \cdot \frac{\left(\phi_{\mathrm{b}}\right)^{\frac{3}{2}}}{F_{\mathrm{ox}}}\right)
$$

where, $A, B$, and $F_{\mathrm{ox}}$ are given as follows:

$$
\begin{aligned}
& A=\frac{q^{3}}{8 \pi h} \\
& F_{\mathrm{ox}}=\frac{V_{\mathrm{ox}}}{t_{\mathrm{ox}}} \\
& B=\frac{8 \pi}{3}\left(\frac{\sqrt{2 \cdot m_{\mathrm{ox}}^{*}}}{h q}\right)
\end{aligned}
$$

In the above equation, $V_{\text {ox }}$ is the voltage across the dielectric, $\phi_{\mathrm{b}}$ is the effective barrier height, and $m_{\mathrm{ox}}^{*}$ is the effective electron mass in the dielectric layer (hBN). FN tunneling equation mainly predicts that the tunneling current in the presence of a sufficiently high electric field, depends exponentially on the barrier height to the power $3 / 2$. However, the FN equation is only applicable to the triangular tunnel barrier, which mainly occurs when $V_{\mathrm{ox}}>\phi_{\mathrm{b}}$. In the current case, we are mainly interested in the low voltage regime, i.e., $V_{\mathrm{ox}}<\phi_{\mathrm{b}}$, where most current flow across hBN through direct tunneling. Most recently, Lee and $\mathrm{Hu}$ proposed a modified FN model to calculate the direct tunneling current $\left(J_{i}\right)(\text { Eq. 10) })^{25}$.

$$
J_{i}=\frac{A}{q \varepsilon_{\mathrm{ox}} \phi_{i, \mathrm{ox}}} \cdot C\left(V, V_{\mathrm{ox}}, t_{\mathrm{ox}}, \phi_{i, \mathrm{ox}}\right) \cdot \exp \left\{-\frac{B\left(\phi_{i, \mathrm{ox}}\right)^{\frac{3}{2}}}{\left|F_{\mathrm{ox}}\right|} \cdot\left[1-\left(1-\frac{\left|V_{\mathrm{ox}}\right|}{\phi_{i, \mathrm{ox}}}\right)^{\frac{3}{2}}\right]\right\}
$$

The most significant difference between Eqs. (9) and (10) is that they introduce a correction factor $C\left(V, V_{\mathrm{ox}}, t_{\mathrm{ox}}, \phi_{i, \mathrm{ox}}\right)$ given by:

$$
C\left(V, V_{\mathrm{ox}}, t_{\mathrm{ox}}, \phi_{i, \mathrm{ox}}\right)=\exp \left[-\frac{20}{\phi_{i, \mathrm{ox}}}\left(\frac{\left|V_{\mathrm{ox}}\right|-\phi_{i, \mathrm{ox}}}{\phi_{i, \mathrm{ox}}}+1\right)^{a_{i, \mathrm{ox}}} \cdot\left(1-\frac{V_{\mathrm{ox}}}{\phi_{i, \mathrm{ox}}}\right)\right] \cdot \frac{V}{t_{\mathrm{ox}}} \cdot N
$$

where, $i(=\mathrm{ECB}$ or $\mathrm{HVB})$ are the indexes to define tunneling of an electron from the conduction band ( $i=\mathrm{ECB}$ ) or tunneling of holes from the valence band ( $i=$ HVB), $N$ is the density of carriers in the inversion or accumulation layer, and $a_{i, \mathrm{ox}}$ is a fitting parameter. The exponential term associated with the correction factor accounts for secondary effects and only affects the curvature of tunneling characteristics. But, one of the benefits of using Lee and $\mathrm{Hu}$ 's equation is that it directly correlates the tunneling current with the accumulation and inversion of carriers, and the conduction and valence band offsets of $\mathrm{i}-\mathrm{InP} / \mathrm{hBN}$ (please see Fig. 6). Overall, Eqs. (9) and (10) respectively show that the tunneling current at any given voltage is inversely proportional to the thickness of the dielectric layer and band offsets, and is directly proportional to the charge carrier density at the dielectric/ semiconductor interface $(\mathrm{hBN} / \mathrm{i}-\mathrm{InP})$. Therefore, in the present

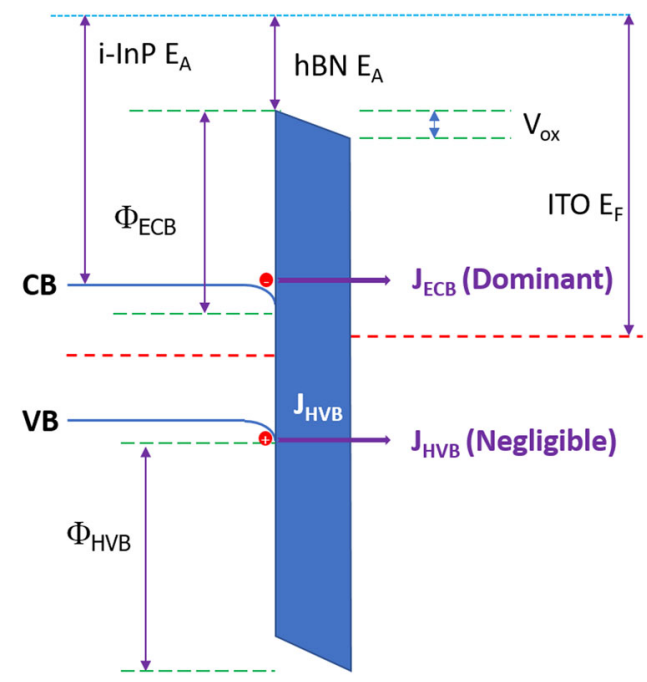

Fig. 6 Depiction of direct tunneling mechanism under interface charge carrier accumulation and depletion. A band diagram depicting the direct quantum mechanical tunneling from i-InP to ITO. Primarily two tunneling components can be calculated using Eq. (10): electron tunneling current from the conduction band $\left(J_{E C B}\right)$, and hole tunneling from the valence band $\left(J_{\mathrm{HVB}}\right) . \phi_{\mathrm{ECB}}$ is the barrier height for electron tunneling from the $\mathrm{i}-\mathrm{InP}$ conduction band with ITO, and $\phi_{\mathrm{HVB}}$ is the barrier height for holes tunneling from the i-InP valence band to ITO. Because $\phi_{\mathrm{ECB}}<\phi_{\mathrm{HVB}}$, and there is an accumulation of electrons at the interface, $J_{\mathrm{ECB}} \gg J_{\mathrm{HVB}}$.

scenario, a thinner hBN layer provides higher $J_{\mathrm{sc}}$ compared to thicker hBN films. At the same time, with increased thickness of the hBN layer, the tunneling current of holes (minority carrier) will also be reduced along with electrons, which will reduce the front contact recombination and lower the dark current, leading to a higher $V_{\text {oc }}$ for thicker hBN compared to a thinner film.

In addition to the passivation layer, hBN may also be acting as a selective contact. Previously, tunnel barriers have also been used as selective contacts ${ }^{26-28}$. For solar cells using dielectric as selective contact, Peibst et al. showed that under first-order approximation, the contact resistance $\rho_{\mathrm{c}}$ (determined by majority carrier flow), and the dark current $J_{0}$ (determined by minority carrier flow) are proportional to the transmission of electrons and holes ${ }^{25}$. Assuming a rectangular dielectric barrier (as shown in Fig. 6) and ignoring the image charge effects, we can write the ratio for electrons and holes tunnel probabilities as follows ${ }^{26}$ :

$$
\frac{T_{\mathrm{e}}}{T_{\mathrm{h}}} \propto \frac{\exp \left\{-t_{\mathrm{ox}} \sqrt{\frac{2 m_{\mathrm{ox}, \mathrm{e}}^{*}}{\hbar^{2}} \phi_{\mathrm{ECB}}}\right\}}{\exp \left\{-t_{\mathrm{ox}} \sqrt{\frac{2 m_{\mathrm{ox}, \mathrm{h}}^{*}}{\hbar^{2}} \phi_{\mathrm{HVB}}}\right\}}
$$

In the above equation, $T_{\mathrm{e}}$ and $T_{\mathrm{h}}$ are electrons and holes tunneling probabilities, respectively, $t_{\mathrm{ox}}$ is the thickness of the dielectric layer, $m_{\mathrm{ox}, \mathrm{e}}^{*}$ and $m_{\mathrm{ox}, \mathrm{h}}^{*}$ are the effective mass of electrons and holes in the dielectric, respectively. The mass of electrons and holes in 2D hBN is similar at $\sim 0.54 \mathrm{~m}_{0}$ (ref. ${ }^{29}$ ). Therefore, for a given thickness of hBN, selectivity toward electrons or holes is solely decided by the barrier height. Therefore, when there is an accumulation of electrons at the semiconductor/hBN interface (as in the current case), the barrier height for electron tunneling reduces, and hBN acts as an electron selective. In contrast, when there is an accumulation of holes at the interface, it will become selective to holes. This is why hBN has previously been reported to act as both electron and hole selective contacts depending on whether there is of electron or hole accumulation at the hBN/ semiconductor interface ${ }^{30}$. 


\section{METHODS}

hBN growth and characterization

The hBN films were grown on commercially available 2 in. sapphire substrate using MOCVD, as described in ref. ${ }^{14}$. The thickness of the films was controlled by varying the growth time. Although several different thicknesses of $h B N$ were investigated for passivation, to keep our discussion concise, here we only discuss the results from two different $\mathrm{hBN}$ films with a thickness of $\sim 1.65$ and $\sim 2.15 \mathrm{~nm}$ corresponding to five and seven monolayers of $\mathrm{hBN}$ (also referred to as $5 \mathrm{ML}$ and $7 \mathrm{ML}$ ), respectively.

For the passivation of the InP surface, $\mathrm{cm}$-size hBN films were transferred from sapphire to InP using the methods described in ref. ${ }^{20}$. First, the sapphire substrate (with $\mathrm{hBN}$ ) was broken into $\mathrm{cm}$-size pieces. Thereafter, PMMA was spin-coated onto the hBN/sapphire substrate and baked on a hot plate to remove excess solvent. The $\mathrm{hBN}$ layer was then floated in a $2 \% \mathrm{HF}$ acid bath. The acid wetted the hBN-sapphire interface and etched the sapphire substrate laterally. After a short time, the hBN layer separated from the sapphire and floated on the surface of the HF bath. Subsequently, the floating hBN-PMMA stack was fished out of the solution and washed several times in deionized (DI) water to remove any residual HF. The hBN-PMMA was then carefully refloated on the surface of a DI water bath and then transferred onto the InP substrate. The samples were allowed to dry under ambient for a few hours. Finally, PMMA was removed in acetone. Using the same process, the hBN-PMMA stack was also transferred on to a planar $\mathrm{SiO}_{2} /$ Si substrate for further characterization. After removing the PMMA layer, the transferred hBN films were characterized using AFM and Raman spectroscopy. Finally, cross-sectional TEM lamella was prepared by focused ion beam milling using FEl's Helios 600 NanoLab, and high-resolution TEM was performed with a JEOL $2100 \mathrm{~F}$ instrument.

\section{Device fabrication and characterization}

A 3D schematic and the cross-sectional TEM images of the solar cell are shown in Fig. 1. It consists of back ohmic contact of low resistance, a $\mathrm{p}^{+}-\ln \mathrm{P}$ base, an $\mathrm{i}-$ InP absorber layer, a hBN layer, an ITO layer, and a metal contact over ITO. The device fabrication started with the epilayer growth of 2 microns thick i-InP on a $\mathrm{p}^{+}-\mathrm{InP}$ wafer. Next, a $\mathrm{Zn} / \mathrm{Au}(20 \mathrm{~nm} / 100 \mathrm{~nm})$ metal contact layer was evaporated on the back of the $\mathrm{p}^{+}-\mathrm{InP}$ substrate, followed by annealing at $400{ }^{\circ} \mathrm{C}$ in forming gas $\left(5 \% \mathrm{H}_{2}: 95 \% \mathrm{~N}_{2}\right)$ for 40 min to create a low resistance back contact. Next, MOCVD-grown hBN was transferred onto the InP substrate, as described above. The hBN adhered to the InP through van der Waals bonding and remains unaffected during further processing of the samples. Thereafter, ITO was sputter-deposited on top of the hBN using conditions reported in ref. ${ }^{5}$ Finally, a 500 -nm thick silver is evaporated through a shadow mask to form the top contact. For comparison, an InP solar cell without hBN layer was also fabricated in parallel under identical processing conditions.

The device characterization started with the confirmation of the successful transfer of $\mathrm{hBN}$ on InP using XPS. To understand the band bending at the interface of ITO/hBN/i-InP, UPS measurements were performed on $\mathrm{i}-\mathrm{InP}, \mathrm{hBN} / \mathrm{i}-\mathrm{InP}$, and ITO. The IV characteristics of the devices were obtained using an Oriel solar simulator and IV test station, under the 1-sun @ air mass $1.5 \mathrm{G}$ (at $25^{\circ} \mathrm{C}$ ) and in the dark. Before every measurement, the solar simulator was calibrated to 1-sun @ air mass $1.5 \mathrm{G}$ (at $25^{\circ} \mathrm{C}$ ), using a standard test sample provided by the manufacturer. The dark IV curve was fitted using the double diode equation to study the effect of hBN passivation. The algorithm used for fitting was based on the work of Suckow et al. ${ }^{21,22}$. The EQE of the sample was measured at room temperature using a quantum efficiency setup from Oriel. Similar to the solar simulator, the EQE setup was calibrated using a commercially available test sample obtained from the manufacturer.

\section{DATA AVAILABILITY}

The data used in this study are available upon request from the corresponding author V.R.

Received: 19 March 2020; Accepted: 2 December 2020; Published online: 08 January 2021

\section{REFERENCES}

1. Raj, V., Tan, H. H. \& Jagadish, C. Non-epitaxial carrier selective contacts for III-V solar cells: a review. Appl. Mater. Today 18, 100503 (2019).

2. Raj, K. Jain. InP solar cell with window layer. USA patent, US5322573 (1994)
3. Connolly, J. P. \& Mencaraglia, D. in Materials Challenges: Inorganic Photovoltaic Solar Energy, 209-246 (The Royal Society of Chemistry, Cambridge, 2015).

4. Mar, J. M. State-of-the-art of III-V solar cell fabrication technologies, device designs and applications. in Advanced Photovoltaic Cell Design, Ch. 1, 1-8 (EN548, 2004).

5. Raj, V. et al. Indium phosphide based solar cell using ultra-thin $\mathrm{ZnO}$ as an electron selective layer. J. Phys. D Appl. Phys. 51, 395301 (2018).

6. Robertson, J., Guo, Y. \& Lin, L. Defect state passivation at III-V oxide interfaces for complementary metal-oxide-semiconductor devices. J. Appl. Phys. 117, 112806 (2015).

7. Lin, L. \& Robertson, J. Defect states at III-V semiconductor oxide interfaces. Appl. Phys. Lett. 98, 082903 (2011).

8. Zhou, L. et al. Brief review of surface passivation on III-V semiconductor. Crystals 8, 226 (2018).

9. Raj, V., Fu, L., Tan, H. H. \& Jagadish, C. Design principles for fabrication of InPbased radial junction nanowire solar cells using an electron selective contact. IEEE J. Photovolt. 9, 980-991 (2019).

10. Raj, V., Vora, K., Fu, L., Tan, H. H. \& Jagadish, C. High-efficiency solar cells from extremely low minority carrier lifetime substrates using radial junction nanowire architecture. ACS Nano 13, 12015-12023 (2019).

11. Hui, F. et al. On the use of two dimensional hexagonal boron nitride as dielectric. Microelectron. Eng. 163, 119-133 (2016).

12. Cinzia C. F. W. 2D Materials for Nanoelectronics (CRC Press, Boca Raton, 2016).

13. Shanmugam, M., Jacobs-Gedrim, R., Durcan, C. \& Yu, B. 2D layered insulator hexagonal boron nitride enabled surface passivation in dye sensitized solar cells. Nanoscale 5, 11275-11282 (2013).

14. Chugh, D. et al. Flow modulation epitaxy of hexagonal boron nitride. 2D Mater. 5 045018 (2018).

15. Cho, A.-J. \& Kwon, J.-Y. Hexagonal boron nitride for surface passivation of twodimensional van der Waals heterojunction solar cells. ACS Appl. Mater. Interfaces 11, 39765-39771 (2019).

16. Kalita, G., Kobayashi, M., Shaarin, M. D., Mahyavanshi, R. D. \& Tanemura, M. Schottky barrier diode characteristics of graphene-GaN heterojunction with hexagonal boron nitride interfacial layer. Phys. Status Solidi A 215, 1800089 (2018).

17. $\mathrm{Li}, \mathrm{X}$. et al. Graphene/h-BN/GaAs sandwich diode as solar cell and photodetector. Opt. Express 24, 134-145 (2016).

18. Li, Q., Zhou, Q., Shi, L., Chen, Q. \& Wang, J. Recent advances in oxidation and degradation mechanisms of ultrathin $2 \mathrm{D}$ materials under ambient conditions and their passivation strategies. J. Mater. Chem. A 7, 4291-4312 (2019).

19. Shanmugam, M., Jain, N., Jacobs-Gedrim, R., Xu, Y. \& Yu, B. Layered insulator hexagonal boron nitride for surface passivation in quantum dot solar cell. Appl. Phys. Lett. 103, 243904 (2013).

20. Chugh, D., Jagadish, C. \& Tan, H. Large-area hexagonal boron nitride for surface enhanced raman spectroscopy. Adv. Mater. Technol. 4, 1900220 (2019).

21. Khanna, A. et al. A fill factor loss analysis method for silicon wafer solar cells. IEEE J. Photovolt. 3, 1170-1177 (2013).

22. Green, M. A. Accuracy of analytical expressions for solar cell fill factors. Sol. Cells $\mathbf{7}$ 337-340 (1982).

23. Terman, L. M. An investigation of surface states at a silicon/silicon oxide interface employing metal-oxide-silicon diodes. Solid State Electron. 5, 285-299 (1962).

24. Park, J. H. et al. Defect passivation of transition metal dichalcogenides via a charge transfer van der Waals interface. Sci. Adv. 3, e1701661 (2017).

25. Wen-Chin, L. \& Chenming, H. Modeling gate and substrate currents due to conduction- and valence-band electron and hole tunneling [CMOS technology]. In 2000 Symposium on VLSI Technology. Digest of Technical Papers (Cat. No.00CH37104) 198-199 (IEEE, Honolulu, HI, 2000).

26. Peibst, R. et al. Working principle of carrier selective poly-Si/c-Si junctions: is tunnelling the whole story? Sol. Energy Mater. Sol. 158, 60-67 (2016).

27. Rienäcker, $M$. et al. Junction resistivity of carrier-selective polysilicon on oxide junctions and its impact on solar cell performance. IEEE J. Photovolt. 7, 11-18 (2017).

28. Wietler, T. F. et al. Pinhole density and contact resistivity of carrier selective junctions with polycrystalline silicon on oxide. Appl. Phys. Lett. 110, 253902 (2017).

29. Cao, X. K., Clubine, B., Edgar, J. H., Lin, J. Y. \& Jiang, H. X. Two-dimensional excitons in three-dimensional hexagonal boron nitride. Appl. Phys. Lett. 103, 191106 (2013).

30. Chu, D., Lee, Y. H. \& Kim, E. K. Selective control of electron and hole tunneling in 2D assembly. Sci. Adv. 3, e1602726 (2017).

\section{ACKNOWLEDGEMENTS}

This work is supported by the Australian Research Council through the DiscoveryProject grants. Access to the epitaxial growth and fabrication facilities is made possible through the support of the Australian National Fabrication Facility, ACT Node. 


\section{AUTHOR CONTRIBUTIONS}

L.E.B. and M.M.S. performed CV measurement and analysis. L.L. and F.K. performed FIB and TEM, respectively. D.H.M., H.H.T., and C.J. supervised the project. V.R. wrote the manuscript with contribution from all the authors. All authors have given approval to the final version of the manuscript.

\section{COMPETING INTERESTS}

The authors declare no competing interests.

\section{ADDITIONAL INFORMATION}

Correspondence and requests for materials should be addressed to V.R. or H.H.T.

Reprints and permission information is available at http://www.nature.com/ reprints
Publisher's note Springer Nature remains neutral with regard to jurisdictional claims in published maps and institutional affiliations.

(i) Open Access This article is licensed under a Creative Commons c. Attribution 4.0 International License, which permits use, sharing, adaptation, distribution and reproduction in any medium or format, as long as you give appropriate credit to the original author(s) and the source, provide a link to the Creative Commons license, and indicate if changes were made. The images or other third party material in this article are included in the article's Creative Commons license, unless indicated otherwise in a credit line to the material. If material is not included in the article's Creative Commons license and your intended use is not permitted by statutory regulation or exceeds the permitted use, you will need to obtain permission directly from the copyright holder. To view a copy of this license, visit http://creativecommons. org/licenses/by/4.0/.

(c) The Author(s) 2021 